\section{Effects of oxidative stress on generation of age in senile myocardial infraction patients}

\author{
Anjuman Gul,' M. Ataur Rahman, ${ }^{2}$ \\ Syed Nazurl Hasnain,' Sadaf Hamid ${ }^{3}$ \\ 'Department of Biochemistry, College \\ of Medicine, Qaseem University Kingdom \\ of Saudi Arabia; \\ ${ }^{2}$ H.E.J. Research Institute of Chemistry, \\ University of Karachi, Pakistan; \\ ${ }^{3}$ Department of Anatomy, Sindh Medical \\ college, Dow University, Pakistan
}

\section{Abstract}

Type 2 diabetes mellitus have a two- to four fold increased risk of myocardial infarction in senile patients. Oxidative stress has been reported to contribute to aging and myocardial infarction in type 2 diabetic patients. This study aims to determine the effects of oxidative stress on generation of AGE in senile myocardial infraction patients. Human serum samples of normal senile subjects $(n=31)$, senile diabetic patients without myocardial infarction $(n=33)$, senile diabetic patients with myocardial infarction ( $\mathrm{n}=32)$, senile nondiabetic with myocardial infarction $(\mathrm{n}=30)$ and normal young subjects $(\mathrm{n}=31)$ were investigated. The patients were selected on clinical grounds from National Institute of Cardiovascular Disease, Karachi and Jinnah Postgraduate Medical Centre, Karachi, Pakistan. Positive significant correlation was observed between s-AGEs and malondialdehyde in senile diabetic and non-diabetic patients with myocardial infarction. Negative significant correlation was observed between s-AGEs and vitamin-E in senile diabetic and non-diabetic patients with myocardial infarction. However, the malondialdehyde and serum AGEs were found to be significantly $(\mathrm{P}<0.001)$ increased in senile diabetic and non-diabetic patients with and without myocardial infarction as compared with senile control subjects. In contrast to all four senile groups, the serum AGEs was significantly $(\mathrm{P}<0.001)$ lower in young control subjects. Serum vitamin-E was found to be significantly $(\mathrm{P}<0.001)$ decreased in elderly diabetic patients with and without myocardial infarction as compared with senile control subjects. Fasting blood glucose, $\mathrm{HbA1C}$ and serum fructosamine levels were significantly $(\mathrm{P}<0.001)$ increased in senile diabetic patients with and without myocardial infarction as compared with non-diabetic senile patients with myocardial infarction and senile control subjects. This study revealed the effects of oxidative stress on generation of
AGE in senile myocardial infraction patients. AGE as a result of oxidative stress might have a role in the myocardial infarction, which in diabetic patients occurs vigorously as compared with non-diabetic patients with myocardial infarction.

\section{Introduction}

Type 2 (non-insulin dependent) diabetes mellitus, the most prevalent form of the disease, is associated with chronic macrovascular and microvascular complications. Among macrovascular complications myocardial infarction is the leading cause of death among senile patients with and without diabetes. ${ }^{1}$ In the developed world, the risk for myocardial infarction is increased two to fourfold among diabetic patients compared with non-diabetic persons within their corresponding population. ${ }^{2}$ Recently, it has been documented that, among various factors, advanced glycation end products (AGEs), a heterogeneous group of irreversibly modified products formed in excess during aging and diabetes mellitus, play a crucial role in this process. ${ }^{3}$ Hyper glycemia can stimulate non-enzymatic glycation and oxidation of proteins and lipids leading to enhancement the formation of AGEs, which may be involved in the pathogenesis of diabetic vascular diseases. ${ }^{4}$ The presences of AGEs have also been reported in the atherosclerotic plaques and the cross-linking abilities of AGEs may contribute to the increased stiffening of collagen and possibly to vascular hypertrophy. ${ }^{5}$ Accumulation of AGEs with structural alterations result in altered tissue properties that contribute to the reduced susceptibility to catabolism. ${ }^{6}$ Another possible mechanism by which AGEs may contribute to development of atherosclerosis is by activating the transcription nuclear factor $\kappa \beta$ (NF- $\kappa \beta)$ through RAGE binding, resulting in induction of cellular adhesion molecule expression and cytokine activation or through glycoxidation of lipoproteins and increased foam cell formation. $^{\text {? }}$

HbA1C and fructosamine are biomarkers that provide a snapshot of long-term glucose control in diabetes. Glycated serum fructosamine (glycoprotein) reflects glycaemic control over the preceding two weeks because of its shorter half-life. Reactive oxygen species (ROS) generated in oxidative stress from a variety of sources can in turn accelerate the AGE formation. Oxidative stress originates due to an imbalance between the generation of ROS and the antioxidant defense system. Production of ROS depletes antioxidants and antioxidant enzymes, leading to additional ROS accumulation. AGE formation is dependent on oxidative processes and can create ROS
Correspondence: Dr. Anjuman Gul,

Professor of Biochemistry, College of Medicine, Qaseem University, Kingdom of Saudi Arabia

E-mail: anjummurtazagul@yahoo.com

Key words: advanced glycation end products, myocardial infarction, senile, diabetes, aging

Acknowledgement: this work was financially supported by Pakistan Science Foundation grant.

Received for publication: 27 August 2010. Accepted for publication: 30 November 2010.

This work is licensed under a Creative Commons Attribution 3.0 License (by-nc 3.0).

CCopyright A. Gul et al., 2011

Licensee PAGEPress, Italy

Endocrinology Studies 2011; 1:e1

doi:10.4081/es.2011.e1

through the Millard reaction. ${ }^{8}$ One of the most frequently used biomarkers providing an indication of the overall lipid peroxidation level is the plasma concentration of malondialdehyde (P-MDA), one of several byproducts of lipid peroxidation processes. During diabetes, an increased production of ROS and an enhanced concentration of thiobarbituric acid-reactive substances (TBARs) resulting in oxidative stress have been observed. ${ }^{9}$ Previous studies also reported that deficiency of vitamin E may be an independent risk factor of CHD. ${ }^{9}$ There are also studies which reports that serum AGEs increased in diabetic and non-diabetic patients with myocardial infarction, but to our knowledge none of these studies has so far reported the correlation of oxidative stress with AGEs in the senile non-diabetic subjects with myocardial infarction. Moreover, the level of serum AGEs could be considered as a marker for the developments of myocardial infarction in senile diabetic as well as in non-diabetic patients.

\section{Materials and Methods}

\section{Subjects and sample collection}

The study included one hundred fifty seven subjects. Out of them 31 were normal senile subjects, 33 were senile diabetic patients without myocardial infarction, 32 were senile diabetic patients with myocardial infarction, 30 were senile non-diabetic with myocardial infarction and 31 were normal young subjects. The blood samples were collected from the subjects during the period of March 2004 to December 2007. The ethical committee of Ziauddin University approved the protocol and consent of the patients were obtained after the 
nature of the study was fully explained. The senile subjects were selected who were over sixty years of age and young apparently healthy (age ranging from 20-25 yrs) were selected as control subjects. Sex, weight, duration of diabetes, duration of complication in diabetic and non-diabetic patients, type of diabetes and type of treatments received were also recorded. Physical examination including measurement of blood pressure was recorded. Individuals were classified as having diabetes mellitus if any of the following criteria were met. ${ }^{10}$ Fasting serum glucose levels of $7.0 \mathrm{mmol} / \mathrm{L}$ or more, random glucose levels of more than 11.1 $\mathrm{mmol} / \mathrm{L}$, current use of medications prescribed to treat diabetes (e.g. insulin or drugs). Senile patients or those with more than one complication were excluded from the study. Diagnosed cases of myocardial infarction were included in the study on the basis of chest pain, ECG changes i.e. ST elevation and $Q$ wave inversion and biochemical markers i.e. raised levels of troponin T, CKMB, AST and LDH. The patients were selected on clinical grounds from National Institute of Cardiovascular Disease, Karachi and Jinnah Postgraduate Medical Centre, Karachi, Pakistan.

Blood was collected in fasting state after a 10-h overnight fast. Samples were withdrawn by venous puncture and distributed equally into three tubes containing EDTA (for HbA1C), heparin (for glucose estimation) and tube with no anti-coagulant (for serum collection). The samples were then immediately stored on ice until processed. Clotted blood was centrifuged at 1,500 rpm for $30 \mathrm{~min}$ and the serum was separated and frozen at $-70^{\circ} \mathrm{C}$ until analysis. Blood glucose was determined by glucose oxidase method, glycosylated hemoglobin (HbA1C) was determined calorimetrically using HbA1C kit (Bio Systems Reagents and Instruments, Spain). The serum fructosamine was determined calorimetrically using fructosamine kit (Randox, UK). At alkaline pH, nitro-blue tetrazolium is reduced by the action of fructosamine to give rise to the production of formazane, the rate of which is directly proportional to the serum fructosamine concentration. The method of vitamin $\mathrm{E}$ based on the oxidation of xylene-extracted tocopherols of the blood sample by ferric chloride and the pink complex of ferrous ions with bathophenanthroline is measured colorimetrically at $536 \mathrm{~nm} .{ }^{11}$ Malondialdehyde of the serum sample was reacted with thiobarbituric acid to form a pink coloured pigment, the absorbance of which was measured at $535 \mathrm{~nm} .^{12}$

\section{Determination of AGEs}

Pretreatment of the serum samples for AGEs measurement: To $100 \mu \mathrm{L}$ of serum diluted with $100 \mathrm{mM}$ phosphate-buffered saline, $\mathrm{pH} 7.2$ (PBS), $100 \mu \mathrm{L}$ of $0.6 \%$ SDS/10 mM Tris- $\mathrm{HCl}$ saline, pH 7.4 and $5 \mu \mathrm{L}$ of $2 \mathrm{M} \mathrm{NaBH}_{4} / 50 \mathrm{mM}$
$\mathrm{NaOH}$ were added. The mixture was immediately heated at $100^{\circ} \mathrm{C}$ for $10 \mathrm{~min}$. After cooling in ice water $800 \mu \mathrm{L}$ of PBS was added again and the samples were then used for AGE assay.

Generation of AGE-BSA (Bovine serum albumin) standard: AGE-BSA was prepared by incubating $5 \mathrm{~g}$ BSA with glucose $(0.56 \mathrm{M})$ in phosphate buffered saline (PBS) under sterile conditions for sixteen weeks at $37^{\circ} \mathrm{C}$. Samples were dialysed against PBS and stored at $-70^{\circ} \mathrm{C}$, protected from light until used.

The amount of AGE was determined by noncompetitive ELISA using rabbit polyclonal antibodies to AGE (Abcam, UK). ${ }^{13}$ A 96 -wells microplate was coated with $200 \mu \mathrm{L}$ of sample and its corresponding control in $50 \mathrm{mM}$ sodium bicarbonate buffer ( $\mathrm{pH} 9.6)$ and kept at $4^{\circ} \mathrm{C}$ for overnight. After overnight incubation, the wells were washed four times using PBS containing 0.05\% Tween-20 (PBST). Each well was blocked for two hours with blocking buffer, washed four times with PBST and incubated with $200 \mu \mathrm{L}$ of 1:104 diluted anti-AGE antibody for $2 \mathrm{~h}$. After washing wells four times, $200 \mu \mathrm{L}$ of 1:2000 diluted HRP-anti-rabbit immunoglobulins (Abcam, UK) were added to each well and incubated for $2 \mathrm{~h}$. Wells were washed five times and reacted with $200 \mu \mathrm{L}$ of $3,3,5,5$ 'tetramethylbenzidine (TMB) solution was added to each well and incubated for $30 \mathrm{~min}$ and absorbance at $650 \mathrm{~nm}$ was measured. Results are expressed as arbitrary AGE units (1 $\mathrm{mU}$ of AGE corresponds to $4 \mu \mathrm{g}$ of AGE-BSA standard).

\section{Statistical analysis}

Data was analyzed using Statistical Package for Social Sciences (SPSS, v 10.0) (SPSS Inc., Chicago, Ill, USA). The results are presented as mean, \pm standard deviation, \pm standard error of mean. The statistical significance of the difference between two mean of various parameters between different groups was evaluated by one-way analysis of variance (ANOVA). The Bonferroni's post hoc test was used to determine which group mean differs. With this test SPSS automatically adjusts the significant level for the multiple comparisons to avoid spurious significant differences being identified (any values below the level of 0.05 was considered as significant).

\section{Results}

Levels of fasting blood glucose, glycosylated hemoglobin, serum fructosamine, serum advanced glycation end products and malondialdehydes were significantly increased $(\mathrm{P}<0.001)$ in diabetic patients with myocardial infarction as compared with non-diabetic patients with myocardial infarction and senile control subjects. Serum advanced glycation end products were significantly increased $(\mathrm{P}<0.001)$ in normal control subjects as compared with young healthy subjects. Levels of vitamin-E were significantly decreased $(\mathrm{P}<0.001)$ in diabetic patients with myocardial infarction as compared with non-diabetic patients with myocardial infarction and senile control subjects (Table 1). Negative significant correlation was found between serum AGEs and vitamin $\mathrm{E}$ in senile diabetic patients with myocardial infarction ( $\mathrm{r}=-0.87$ ) (Figure 1a), negative significant correlation was found between serum AGEs and vitamin $\mathrm{E}$ in senile non-diabetic patients with myocardial infarction ( $\mathrm{r}=-0.94)$ (Figure 1b), positive significant correlation was found between serum AGEs and malondialdehydes in senile diabetic patients with myocardial infarction $(\mathrm{r}=0.92)$ (Figure 1c), positive significant correlation was found between serum AGEs and malondialdehydes in senile non-diabetic patients with myocardial infarction ( $\mathrm{r}=0.98$ ) (Figure 1d) and negative significant correlation was found between serum malondialdehydes and vitamin $\mathrm{E}$ in senile diabetic patients with myocardial infarction ( $\mathrm{r}=-0.88$ ) (Figure 1e). Positive significant correlation was observed between fasting blood glucose and serum fructosamine $(\mathrm{r}=0.96)$, fasting blood glucose and systolic blood pressure ( $\mathrm{r}=0.91)$, fasting blood glucose and serum AGEs ( $r=0.94)$ and systolic blood pressure and serum AGEs $(r=0.89)$ in diabetic patients with myocardial infarction. Level of malondialdehyde and serum AGEs were significantly increased $(\mathrm{P}<0.001)$ in senile diabetic patients with and without myocardial infarction and senile non-diabetic patients with myocardial infarction as compared with senile control subjects. Serum vitamin-E was found to be significantly $(\mathrm{P}<0.001)$ decreased in senile diabetic patients with and without myocardial infarction as compared with senile control subjects. In addition, s-AGEs in the normal senile subjects as compared with the normal young subjects show the evaluation of the advancement of biological aging on the formation of AGEs. The senile normal subjects showed a significant increase in the AGEs $(\mathrm{P}<0.001)$ as compared with normal young subjects. Fasting blood glucose, HbA1C and serum fructosamine were significantly increased in senile diabetic patients with or without myocardial infarction as compared with senile non-diabetic patients with myocardial infarction and senile control subjects. The increase in the fasting blood glucose level in all senile diabetic patients with and without myocardial infarction correlates significantly with glycosylated hemoglobin and serum fructosamine concentrations. Also, the fasting blood glucose, glycosylated hemoglobin and serum fructosamine were not found to be different in senile diabetic patients with and without myocardial infarction. When compared with age matched normal subjects, the senile 
non-diabetic patients with myocardial infarction showed no significant difference in levels of fasting blood glucose, glycosylated hemoglobin and serum fructosamine.

\section{Discussion}

Diabetes mellitus is one of the major health problem of Pakistan. The number of people with diabetes is increasing due to population growth, aging, urbanization, and increasing prevalence of obesity and lack of physical inactivity. ${ }^{2}$ Diabetics are prone to long-term complications such as myocardial infarction and development of such complications is a major cause of morbidity and mortality and is an ever-increasing burden to healthcare authorities in both developed and developing nations. ${ }^{14}$ Epidemiological studies have confirmed that hyperglycemia is the most important factor in the onset and progress of vascular complications in diabetes. ${ }^{15}$ The formation of AGEs correlates with glycemic control. The AGE hypothesis proposes that accelerated chemical modification of proteins by glucose during hyperglycemia contributes to the pathogenesis of diabetic complications including atherosclerosis. ${ }^{16}$ Glycation has both physiological and pathophysiological significance. Under physiological conditions, glycation can be detected in the ageing process and the reactions are more rapid and more intensive with frequently increased glucose concentrations. ${ }^{17}$ The AGE concept proposes that chemical modification and crosslinking of tissue proteins, lipids and DNA affect their structure and func-
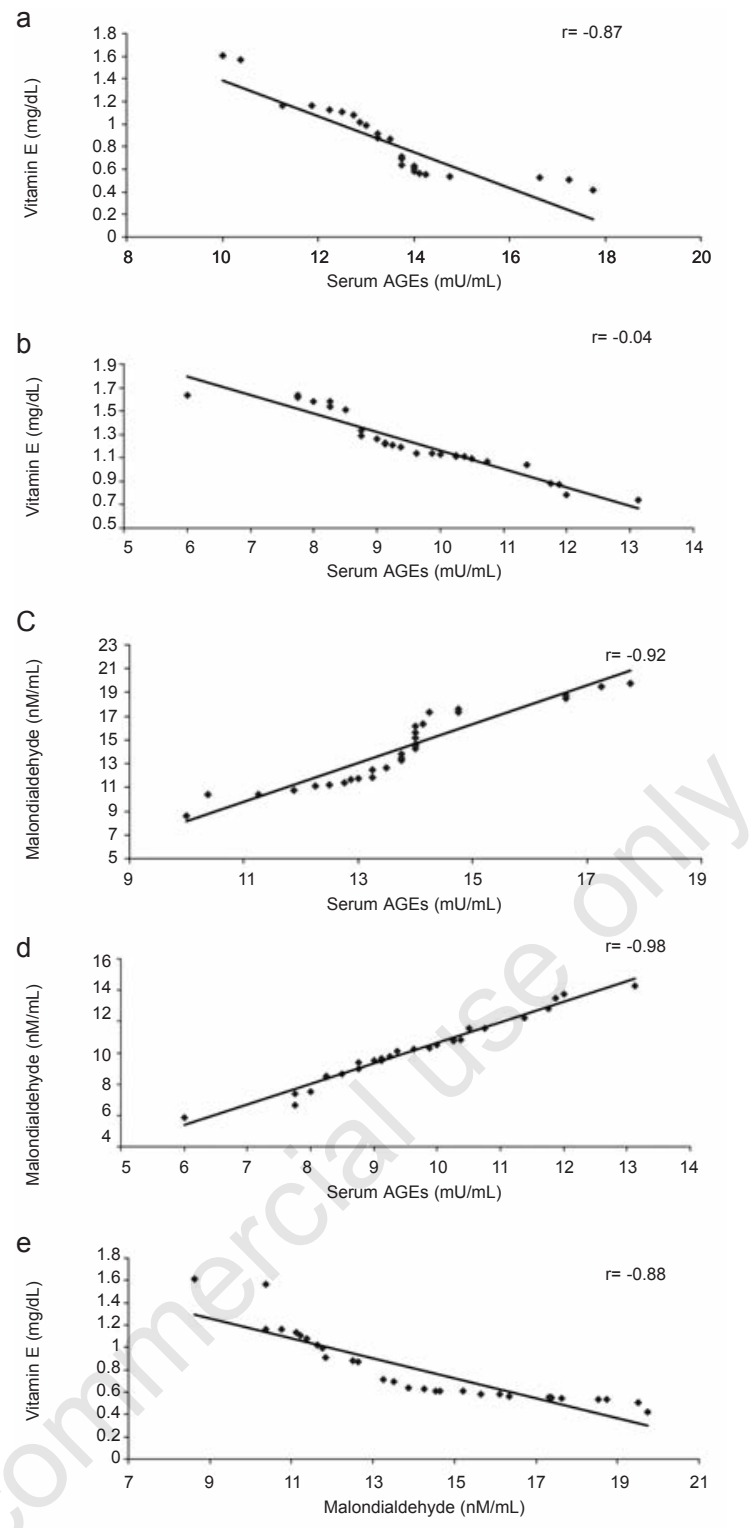

Figure 1. (a) Correlation of serum AGEs vs. vitamin-E in diabetic patients with myocardial infarction. (b) Correlation of serum AGEs vs. vitamin-E in non-diabetic patients with myocardial infarction. (c) Correlation of serum AGEs vs. Malondialdehyde in diabetic patients with myocardial infarction. (d) Correlation of serum AGEs vs. Malondialdehyde in non-diabetic patients with myocardial infarction. (e) Correlation of Malondialdehyde vs. vitamin $E$ in diabetic patients with myocardial infarction.

Table 1. Physical features and blood analysis of young healthy subjects, senile control subjects, senile diabetic patients without myocardial infarction and senile diabetic and non-diabetic patients with myocardial infarction. The values are expressed as mean, \pm standard deviation, \pm standard error of mean. Units and numbers of cases are shown in parentheses.

\begin{tabular}{|c|c|c|c|c|c|}
\hline Parameters & $\begin{array}{l}\text { Young healthy } \\
\text { subjects }^{31}\end{array}$ & $\begin{array}{l}\text { Senile control } \\
\text { subjects }^{31}\end{array}$ & $\begin{array}{l}\text { Senile diabetic } \\
\text { patients without } \\
\text { myocardial } \\
\text { infarction }{ }^{33}\end{array}$ & $\begin{array}{l}\text { Senile diabetic } \\
\text { patients with } \\
\text { myocardial } \\
\text { infarction }\end{array}$ & $\begin{array}{l}\text { Senile non-diabetic } \\
\text { patients with } \\
\text { myocardial } \\
\text { infarction }{ }^{30}\end{array}$ \\
\hline Age (years) & $21.93 \pm 1.59 \pm 0.28$ & $64.19 \mathrm{a} \pm 3.94 \pm 0.70$ & $64.18 \pm 3.31 \pm 0.57$ & $66.00 \pm 4.45 \pm 0.78$ & $65.73 \pm 4.68 \pm 0.85$ \\
\hline $\operatorname{Sex}(F / M)$ & $16 / 15$ & $16 / 15$ & $16 / 17$ & $14 / 18$ & $15 / 15$ \\
\hline Weight (kg) & $59.06 \pm 6.22 \pm 1.11$ & $63.61 \pm 6.82 \pm 1.22$ & $65.66 \pm 8.78 \pm 1.53$ & $66.03 \pm 6.04 \pm 1.07$ & $63.33 \pm 6.90 \pm 1.26$ \\
\hline Height (m) & $1.59 \pm 0.06 \pm 0.01$ & $1.59 \pm 0.05 \pm 0.01$ & $1.59 \pm 0.05 \pm 0.01$ & $1.58 \pm 0.05 \pm 0.01$ & $1.60 \pm 0.06 \pm 0.01$ \\
\hline BMI $\left(\mathrm{kg} / \mathrm{m}^{2}\right)$ & $23.39 \pm 2.70 \pm 0.48$ & $25.22 \pm 2.97 \pm 0.53$ & $26.00 \pm 3.70 \pm 0.64$ & $26.20 c \pm 2.91 \pm 0.51$ & $24.78 \pm 3.12 \pm 0.57$ \\
\hline Systolic BP (mmHg) & $119.51 \pm 6.23 \pm 1.12$ & $121.54 \pm 5.88 \pm 1.05$ & $119.70 \pm 6.83 \pm 1.19$ & $144.53^{\mathrm{b}} \pm 24.27 \pm 4.29$ & $139.16 \mathrm{~b} \pm 24.81 \pm 4.53$ \\
\hline Diastolic BP (mmHg) & $79.19 \pm 5.64 \pm 1.01$ & $83.06 \pm 6.14 \pm 1.10$ & $81.66 \pm 6.08 \pm 1.05$ & $91.56^{\mathrm{b}} \pm 9.45 \pm 1.67$ & $87.33 \mathrm{~b} \pm 9.89 \pm 1.80$ \\
\hline Fasting blood glucose $(\mathrm{mmol} / \mathrm{L})$ & $4.88 \pm 0.50 \pm 0.09$ & $5.09 \pm 0.58 \pm 0.10$ & $7.46^{\mathrm{b}} \pm 1.42 \pm 0.24$ & $8.97 \mathrm{bc} \pm 1.68 \pm 0.29$ & $5.07 \pm 0.67 \pm 0.12$ \\
\hline Glycosylated hemoglobin (HBAlc \%) & $4.81 \pm 0.43 \pm 0.07$ & $4.97 \pm 0.46 \pm 0.08$ & $9.02^{\mathrm{b}} \pm 1.61 \pm 0.28$ & $9.26 \mathrm{bc} \pm 1.52 \pm 0.26$ & $5.09 \pm 0.58 \pm 0.10$ \\
\hline Serum fructosamine $(\mathrm{mmol} / \mathrm{L})$ & $2.10 \pm 0.34 \pm 0.06$ & $2.33 \pm 0.35 \pm 0.06$ & $3.79 \mathrm{~b} \pm 0.66 \pm 0.11$ & $3.74 \mathrm{bc} \pm 0.64 \pm 0.11$ & $2.08 \pm 0.55 \pm 0.10$ \\
\hline Serum-AGEs (mU/mL) & $1.71 \pm 1.42 \pm 0.25$ & $04.97 \mathrm{a} \pm 1.94 \pm 0.34$ & $8.10^{\mathrm{b}} \pm 2.84 \pm 0.49$ & $13.78 \mathrm{bc} \pm 1.71 \pm 0.30$ & $9.55^{\mathrm{b}} \pm 1.51 \pm 0.27$ \\
\hline Malondialdehyde (nM/mL) & - & $3.56 \pm 1.47 \pm 0.26$ & $7.06^{\mathrm{b}} \pm 1.75 \pm 0.30$ & $14.33 \mathrm{bc} \pm 3.04 \pm 0.53$ & $10.03^{\mathrm{b}} \pm 2.01 \pm 0.36$ \\
\hline Vitamin-E (mg/dL) & - & $1.66 \pm 0.33 \pm 0.06$ & $0.89 \mathrm{~b} \pm 0.31 \pm 0.05$ & $0.78 \mathrm{bc} \pm 0.30 \pm 0.05$ & $1.23^{\mathrm{b}} \pm 0.25 \pm 0.04$ \\
\hline
\end{tabular}

aSignificant as compared with young healthy subjects; bsignificant as compared with senile control subjects; csignificant as compared with non-diabetic senile patients with myocardial infarction. 
tion. This in turn contributes to a gradual decline in tissue function and to the pathogenesis of myocardial infarction in diabetic and in nondiabetic patients. ${ }^{18,19}$ AGEs have previously been shown to accumulate in many tissues with age, independently of diabetes. ${ }^{20}$ Since the body does not contain any single enzyme capable of AGE structure degradation, AGEs accumulate during the biological life of proteins on which they had been formed. ${ }^{21}$

In present study, antioxidant defense mechanism by vitamin-E and oxidative stress causing agent by malondialdehyde in senile diabetic and non-diabetic patients with and without myocardial infarction were investigated. Malondialdehyde levels significantly increased $(\mathrm{P}<0.001)$ in senile diabetic patients with or without myocardial infarction and senile nondiabetic patients with myocardial infarction as compared with in senile control subjects. In addition, serum vitamin- $\mathrm{E}$ was found to be significantly decreased $(\mathrm{P}<0.001)$ in senile diabetic patients with and without myocardial infarction as compared with senile control subjects. In the present study, the increased levels of malondialdehyde and decreased vitamin-E clearly show that diabetic patients, irrespective of the gender, were exposed to an increased oxidative stress via lipid peroxidation. This could be due to failure of antioxidant defense system, the ROS accumulates and initiates lipid peroxidation. ${ }^{22}$ Present results are consistent with earlier reports indicating an elevated level of serum lipid peroxide and diminished antioxidant status in diabetic patients. ${ }^{23,24}$ Decreased antioxidant activity and high concentration of lipid peroxidation product may lead to oxidation proteins resulting in myocardial infarction. Previous data shows enhanced ROS levels in diabetes mellitus. ${ }^{25}$ Yan et $a{ }^{26}$ showed that interaction of AGEs with endothelial cells leads to oxidative stress by a receptor-mediated process. AGEs might induce oxidative stress through chemical and cellular mechanisms. In addition, to the monosaccharides, the AGEs have also been reported to be produced from dicarbonyl compounds derived from the Maillard reaction, autoxidation of sugars and other metabollic pathways e.g. glycolysis and this can account for the increase in the s-AGEs in non-diabetic patients with myocardial infarction. ${ }^{27}$ Recent studies have brought new insights into broad derangements in non-enzymatic glycation involving not only carbohydrates but also lipids, present in diabetes, uremia and atherosclerosis. ${ }^{28}$ Increased level of AGEs content in diabetic state was reported earlier. ${ }^{29}$ The results of this study are also being in accordance with these reports. Therefore, it can be speculated that the AGE structures resulting from persisting hyperglycemia are more profusely formed in diabetes and the fact that tissue levels of AGEs correlate with prevailing serum concentrations of glucose, fructoselysine and glycated hemoglobin, points to a role for hyperglycemia, yet there is good evidence that other carbohydrates such as ascorbate, pentoses may act as potent glycating agents. ${ }^{30}$

In addition to the diabetic patients, the serum AGEs was also found to be higher in the senile non-diabetic patients with myocardial infarction as compared with senile diabetic patients without myocardial infarction, however, this increase was not significant. It states that the role of AGEs in diabetic and non-diabetic patients is potentially important because it induces both the structural and functional implications. Environmental conditions can results in the formations of various AGEs by a variety of chemical reactions and the reasons for the formation of such structures in nondiabetic conditions are difficult to explain. Studies have suggested that role of oxidative stress in the formation of AGEs structures, therefore, it might be postulated that reactive oxygen intermediates may accelerate the rate of AGE formation through reactive oxoaldehydes and vice versa, AGEs might induce oxidative stress through chemical and cellular mechanisms. ${ }^{31,32}$ The observations of senile groups increased as compared with that of young normal subjects. This indicates that these changes are correlated with the advancement of the age.

A limitation of this study was the low number of patients and if the study is carried out on a larger sample of patients which is necessary for definite conclusion. Thus the results of this study support the hypothesis that AGEs may have an important role in myocardial infarction, which in diabetic patients occur much earlier than in those without diabetes. This study also revealed that increased oxidative stress associated with AGEs in the senile groups. Taken together the above facts and results it can be postulated that utilization of antioxidant rich food along with low AGEs content diet may be beneficial in delaying myocardial infarction progression particularly in diabetic subjects.

\section{References}

1. Aronson D. Hyperglycemia and the pathobiology of diabetic complications. Adv Cardiol 2008;45:1-16.

2. Saleheen D, Frossard P. CAD risk factors and acute myocardial infarction in Pakistan. Acta Cardiol 2004;59:417-24.

3. Vlassara H, Palace MR. Glycoxidation: the menace of diabetes and aging. Mt Sinai J Med 2003;70:232-41.

4. Soldatos G, Cooper ME. Advanced glycation end products and vascular structure and function. Curr Hypertens Rep 2006;8:472-8.
5. Price CL, Knight SC. Advanced glycation: a novel outlook on atherosclerosis. Curr Pharm Des 2007;13:3681-7.

6. Baynes JW, Thorpe SR. Glycoxidation and lipoxidation in atherogenesis. Free Radical Biol Med 2000; 28:1708-16.

7. Bierhaus A, Chevion S, Chevion M, et al. Advanced glycation end product-induced activation of NF-kB is suppressed by alipoic acid in cultured endothelial cells. Diabetes 1997;46:1481-90.

8. Yamagishi S, Imaizumi T. Diabetic vascular complications: pathophysiology, biochemical basis and potential therapeutic strategy. Curr Pharm Des 2005;11:2279-99.

9. Surekha RH, Srikanth BB, Jharna P, et al. Oxidative stress and total antioxidant status in myocardial infarction. Singapore Med J 2007; 48:137-42.

10. Gabir MM, Roumain J, Hanson RL, et al. The 1997 American Diabetes Association and 1999 World Health Organization criteria for hyperglycemia in the diagnosis and prediction of diabetes. Diabetes Care 2000;23:1108-12.

11. Baker H, Frank O, Angelis B, Feingold S. Plasma tocopherol in man at various times after ingesting free or acetylated tocopherol. Nutr Rep Int 1980;21:531-6.

12. Valenzuela A. The biological significance of malondialdehyde determination in the assessment of tissue oxidative stress. Life Sci 1991;48:301-9.

13. Ono Y, Aoki S, Ohnishi K, et al. Increased serum levels of advanced glycation endproducts and diabetic complications. Diabetes Res Clin Pract 1998;41:131-7.

14. Veiraiah A. Hyperglycemia, lipoprotein glycation, and vascular disease. Angiology 2005;56:431-8.

15. Shera S. Prevalence and prevention. Diabetes Digest 1998;12:7-8.

16. Yamagishi $S$, Matsui $T$, Ueda $S$, et al. Advanced glycation end products (AGEs) and cardiovascular disease (CVD) in diabetes. Cardiovasc Hematol Agents Med Chem 2007;5:236-40.

17. Ulrich P, Cerami A. Protein glycation, diabetes, and aging. Recent Prog Horm Res 2001;56:1-21.

18. Xanthis A, Hatzitolios A, Koliakos G, Tatola V. Advanced glycosylation end products and nutrition-a possible relation with diabetic atherosclerosis and how to prevent it. J Food Sci 2007;72:125-9.

19. Kanauchi M, Tsujimoto N, Hashimoto T. Advanced glycation end products in nondiabetic patients with coronary artery disease. Diabetes Care 2001;24:1620-3.

20. Lingelbach LB, Mitchell AE, Rucker RB, McDonald RB. Accumulation of advanced glycation end products in aging male Fischer 344 rats during long-term feeding of various dietary carbohydrates. J Nutr 
2000;130:1247-55.

21. Yan SF, Yan SD, Herold K, et al. Receptor for advanced glycation end products and the cardiovascular complications of diabetes and beyond: lessons from AGEing. Endocrinol Metab Clin North Am 2006;35: 511-24.

22. Kunitomo M. Oxidative stress and atherosclerosis. Yakugaku Zasshi 2007;127:19972014.

23. Senthil S, Veerappan RM, Ramakrishna Rao M, Pugalendi KV. Oxidative stress and antioxidants in patients with cardiogenic shock complicating acute myocardial infarction. Clin Chim Acta 2004;348:131-7.

24. Su J, Lucchesi PA, Gonzalez-Villalobos RA, et al. Role of advanced glycation end products with oxidative stress in resistance artery dysfunction in Type 2 diabetic mice.
Arterioscler Thromb Vasc Biol 2008; (in press).

25. Schleicher E, Friess U. Oxidative stress, AGE, and atherosclerosis. Kidney Int Suppl 2007;106:S17-26.

26. Yan SD, Schmidt AM, Anderson GM, et al. Enhanced cellular oxidant stress by the interaction of advanced glycation end products with their receptors/binding proteins. J Biol Chem 1994;269:9889-9897.

27. Peppa M, Uribarri J, Vlassara H. The role of advanced glycation end products in the development of atherosclerosis. Curr Diab Rep 2004;4:31-6.

28. Chuyen NV. Toxicity of the AGEs generated from the Maillard reaction: on the relationship of food-AGEs and biological-AGEs. Mol Nutr Food Res 2006;50:1140-9.

29. Ahmed N. Advanced glycation endprod- ucts--role in pathology of diabetic complications. Diabetes Res Clin Pract 2005;67:321.

30. Dyer DG, Blackledge JA, Thorpe SR, Baynes JW. Formation of pentosidine during nonenzymatic browning of protein by glucose: Identification of glucose and other carbohydrates as possible precursors of pentosidine in vivo. J Biol Chem 1991; 266:11654-60.

31. Basta G, Del Turco S, De Caterina R. Advanced glycation end products: implications for accelerated atherosclerosis in diabetes. Recenti Prog Med 2004;95:67-80.

32. Miyata T, Ishikawa N, van Ypersele de Strihou C. Carbonyl stress and diabetic complications. Clin Chem Lab Med 2003; 41:1150-8. 